\title{
Calvarial Tuberculosis With Intracranial Tuberculomas: A Rare Association
}

\author{
Rajendra Singh Jain a, , Swayam Prakasha, Tarun Mathur ${ }^{\mathrm{a}}$, \\ Rahul Handa ${ }^{a}$, Kadam Nagpal ${ }^{a}$
}

\begin{abstract}
Tuberculosis is an infectious disease caused by the acid-fast bacilli Mycobacterium tuberculosis that usually affect the lungs although it may affect any organ. Though incidence of tuberculosis is on rise especially in third world countries like India, tuberculous osteitis of the cranial bones, also known as calvarial tuberculosis, is an uncommon manifestation of disseminated tuberculosis. Calvarial tuberculosis as primary tubercular infection is very rare and rarer still is its association with tuberculomas. We report a case with multiple osteolytic cranial lesions and tuberculomas without focus of infection elsewhere in the body.
\end{abstract}

Keywords: Calvarial; Tuberculoma; Generalized tonic clonic seizure; Antituberculous treatment; Osteolytic

\section{Introduction}

Though tuberculosis is very common especially in the developing countries like India, in both immunocompetent and immunocompromised set of patients, tuberculosis of flat bones of skull is a rare entity and its association with tuberculomas is rarer still, with only a few reports in literature. The overall incidence of skull tuberculosis is approximately $1 / 10,000$ cases of tuberculosis $[1,2]$. Here, we are reporting a rare case of calvarial tuberculosis with multiple intracranial tuberculomas without focus of infection elsewhere.

\section{Case Report}

A 37 years old right-handed man, non-hypertensive, non-

Manuscript accepted for publication June 12, 2013

${ }^{a}$ Department of Neurology, SMS Medical College Hospital, Jaipur, Rajasthan, India

${ }^{b}$ Corresponding author: Rajendra Singh Jain, Department of Neurology, SMS Medical College Hospital, Jaipur, Rajasthan, India.

Email: drrsjain@yahoo.com

doi: http://dx.doi.org/10.4021/jnr210w diabetic, without any significant personal or family history presented with recurrent swellings with creamy sticky discharge over right side of the scalp for nineteen years. Swellings kept on recurring despite surgical excision and antibiotic treatments. However, there were no other symptoms until ten years back when he developed an episode of generalized tonic clonic seizure (GTCS). At this stage contrast CT head showed multiple ring enhancing lesions, and based on that he was started on antituberculous treatment (ATT) and Phenytoin. He stopped treatment after two months due to alleged poor tolerance, leading to recurrence of seizure four months later. He was again started on ATT and carbamazepine (CBZ) $600 \mathrm{mg}$ per day in divided doses. However, he did not take ATT at all due to apprehension of intolerance but continued CBZ for two and a half years and remained seizure free. He approached us at this stage with generalized headache alongwith intermittent increase in intensity especially in morning hours, in addition to secreting scalp lesions.

Neurological examination including fundus was normal. Multiple, small, nodular, fluctuant, nontender swellings over right parietal, temporal and occipital scalp region were present. ESR was $80 \mathrm{~mm}$. HIV and VDRL tests were negative. CSF showed 25 cells (P 30\%, L 70\%), normal sugar (55 mg/ $\mathrm{dL})$, and raised protein $(98 \mathrm{mg} / \mathrm{dL})$. TB-PCR and India ink staining were negative. Mantoux test (MT) was strongly positive $(25 \times 20 \mathrm{~mm})$. X-ray chest was normal.

MRI brain showed osteolytic lesions with soft tissue components in right temporal, parietal and occipital bones causing mass effect over the underlying brain parenchyma. Multiple heterogeneous signals in both occipitals, parietal and right temporal cortices on T2WI surrounded by hyperintense vasogenic edema were seen. Thrombosis of right sigmoid and transverse sinuses was also noted. Contrast administration revealed diffuse bony and dural enhancement and enhancement of occipital and parietal cortical lesions. MR spectroscopy showed reduced NAA and creatinine peaks, large lipid lactate peak (at $0.9 \mathrm{ppm}$ ), and non elevated choline peak suggestive of tubercular etiology. Histopathological examination of deep mastoid bone showed fragments of sclerotic and dead bone with focal chronic inflammation, consistent with chronic osteomyelitis. 


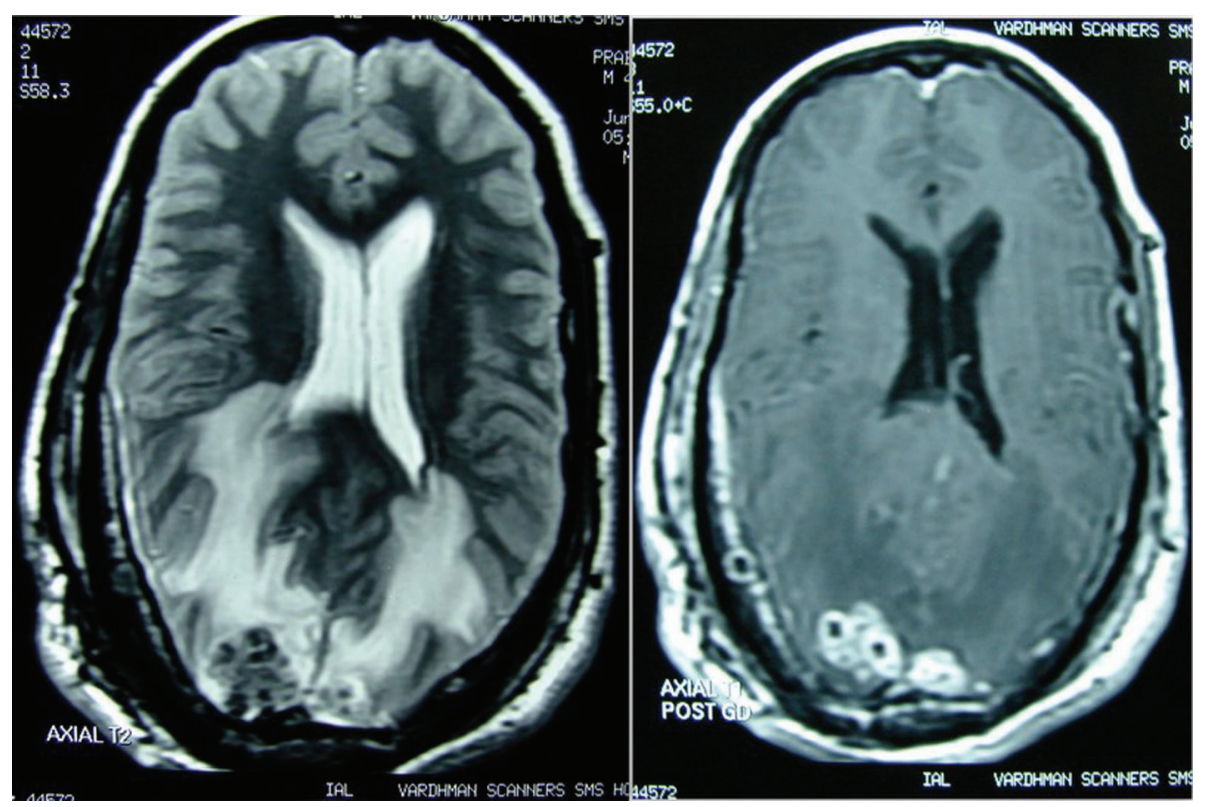

Figure 1. Before starting ATT, osteolytic lesions with soft tissue components in right parieto-occipital region and multiple parenchymal granulomas in both occipital and parietal cortices with marked perilesional edema on $\mathrm{T} 2 \mathrm{Wl}$ and diffuse dural and lesional post contrast enhancement.

Based on the CT, contrast MRI and MRS brain findings, raised ESR and strongly positive MT, a diagnosis of calvarial tuberculosis with intracranial tuberculomas was made and ATT (3 RHZES + RH) started. Patient showed marked symptomatic improvement and follow-up MRI, done seven months after starting ATT, showed marked reduction in size and number of parenchymal granulomas in right parietal, temporal and bilateral occipital lobes with marked regression of edema in bilateral hemispheres. There was no evidence of new lesion. The edema and signal abnormalities in affected calvarial bones like right squamous temporal, petrous, mastoid and occipital bones disappeared with evidence of marked sclerosis in affected bones and thickening of cortex without cortical erosion or breach, suggestive of

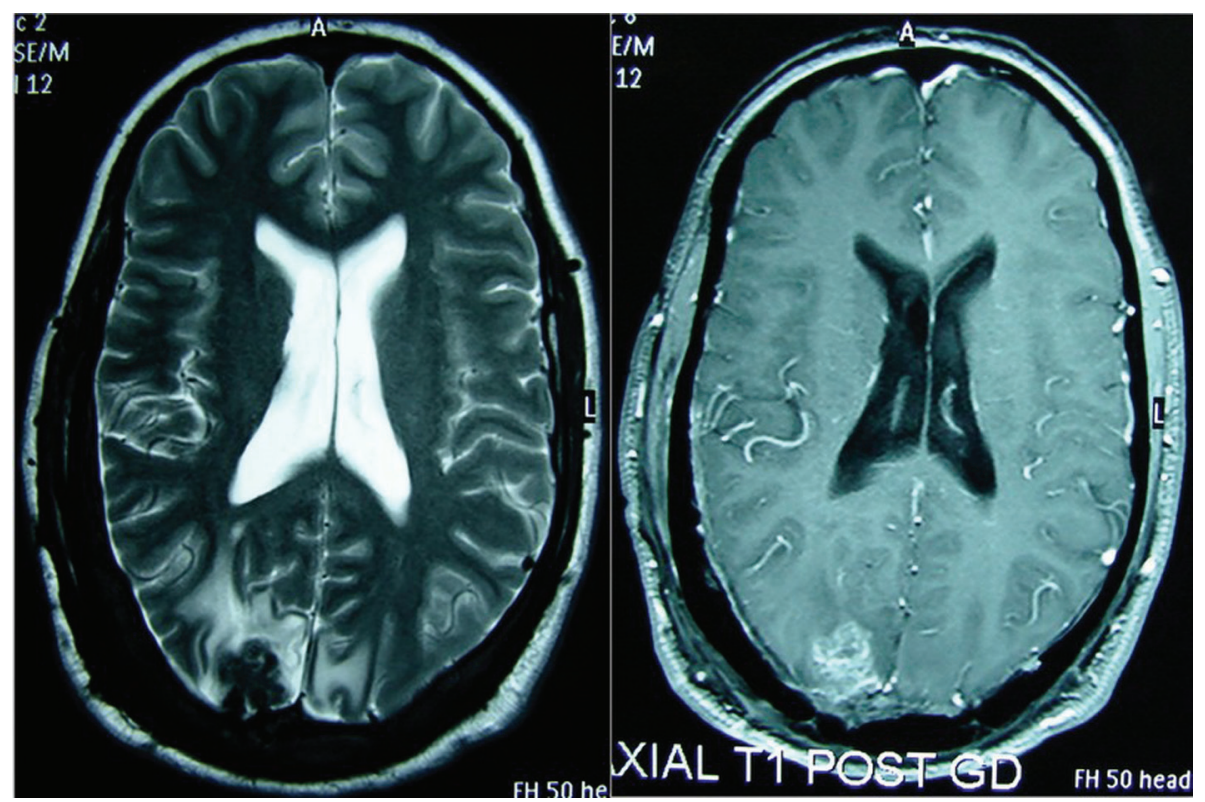

Figure 2. Seven months after starting ATT, marked reduction in size and number of parenchymal granulomas and surrounding edema with marked sclerosis of the affected calvarial bones compared to Figure 1. 
healing disease.

\section{Discussion}

Isolated calvarial tuberculosis is rare due to paucity of lymphatics [1] and little cancellous component in the calvarial bones [3]. The frontal and parietal bones, having greater area of diploic space and cancellous bones are more vulnerable $[4,5]$. However, in our case temporal and occipital bones were affected the most. Though it can occur through hematogenous spread from some primary focus like pulmonary tuberculosis, cervical lymphadenitis or tubercular osteomyelitis of other bones; in the absence of any other focus it could be considered as primary focus itself [6]. Further, association of calvarial tuberculosis with tuberculomas is rarer still but there are reports documenting spread of calvarial tuberculosis to meninges and parenchyma leading to tuberculoma formation of respective structures $[2,7]$.

The radiological picture can vary, with either an osteolytic or a sclerotic variety being seen; the osteolytic type itself can be circumscribed or of the spreading type $[3,8$, $9,10]$. The CT picture of tuberculous osteomyelitis is not very specific. Pyogenic osteomyelitis, calvarial metastases, myeloma, hemangioma, giant cell tumor or even an aneurysmal bone cyst and Langerhans cell histiocytosis form the important differential diagnoses [1, 3, 9]. However MRI with contrast studies combined with MRS are more informative.

Confirmation of diagnosis is by isolation of tubercle bacilli, however, it is not always feasible. In the absence of this, clinical history of recurrent nontender nodular fluctuating scalp swellings with creamy sticky discharge, MRI brain suggestive of osteolytic lesions of the skull bones with multiple intracranial granulomas, MRS showing giant lactate peak at $0.9 \mathrm{ppm}$ and histological examination suggestive of chronic osteomyelitis in our case pointed towards a tubercular aetiology and it was well supported by a satisfactory therapeutic response with ATT $[3,10,11]$.

So to conclude, this case is an eye opener in the sense that tuberculosis of the skull should be suspected in all cases of secreting lesions of the scalp that fail to respond to standard antibiotic therapy and surgical measures [5]. Though rare, calvarial tuberculosis can occur in the absence of focus elsewhere in the body. Isolation of tubercle bacilli is the gold standard criteria for diagnosis, yet it is not always feasible and therefore, MRI and MRS brain findings in the appropriate clinical setting can be an important aetiological clue especially if supported aptly by a satisfactory therapeutic response as in our case.

\section{References}

1. Myoung Soo Kim (2012). Skull Osteomyelitis, Osteomyelitis, Prof. Mauricio S. Baptista (Ed.), ISBN: 978953-51-0399-8, InTech, Available from: http://www.intechopen.com/books/osteomyelitis/skull-osteomyelitis.

2. Strauss DC. Tuberculosis of flat bones of the vault of the skull. Surg Gynecol Obstet 1933; 57: pp 384-398.

3. Bhandari B, Mandowara SL, Joshi H. Tubercular osteomyelitis of skull. Indian J Pediatr. 1981;48(390):113115.

4. Meng CM, WU YK. Tuberculosis of the flat bones of the vault of the skull. JBone Joint Surg 1942;34:341-53.

5. Raut AA, Nagar AM, Muzumdar D, Chawla AJ, Narlawar RS, Fattepurkar S, Bhatgadde VL. Imaging features of calvarial tuberculosis: a study of 42 cases. AJNR Am J Neuroradiol. 2004;25(3):409-414.

6. Gupta K.B., Tandon Sanjeev, Sen Rajeev, Kalra Rajnish; Tuberculosis of flat bones of the vault of skull- A case repot, Ind. J. Tub., 1998, 45, 231.

7. Sinh G, Pandya SK, Dastur DK. Pathogenesis of unusual intracranial tuberculomas and tuberculous spaceoccupying lesions. J Neurosurg. 1968;29(2):149-159.

8. Jadhav RN, Palande DA. Calvarial tuberculosis. Neurosurgery. 1999;45(6):1345-1349; discussion 1349-1350.

9. Samson SK, Kulkarni V, Chacko AG. An unusual presentation of calvarial tuberculosis. Postgrad Med J. 2002;78(917):184, 188-189.

10. Patankar T, Varma R, Krishnan A, Prasad S, Desai K, Castillo M. Radiographic findings in tuberculosis of the calvarium. Neuroradiology. 2000;42(7):518-521.

11. Ravigilione MC, O’Brien RJ. Tuberculosis. In: Harrison's Principles of Internal Medicine. Ed. Fauci SA, Longo DL, 14th edition, 1996, McGraw Hill publishers, Newyork, pp 1004-1014. 